\title{
Explaining the antioxidant activity of some common non-phenolic components of essential oils
}

\author{
Andrea Baschieri, ${ }^{1}$ Majlinda Daci Ajvazi, ${ }^{2, *}$ Judith Laure Folifack Tonfack,${ }^{1}$ Luca Valgimigli, ${ }^{1}, *$ \\ and Riccardo Amorati ${ }^{1, *}$ \\ ${ }^{1}$ University of Bologna, Department of Chemistry "G. Ciamician", Via S. Giacomo 11, 40126 \\ Bologna, Italy.E-mail: luca.valgimigli@unibo.it; riccardo.amorati@unibo.it \\ ${ }^{2}$ University of Prishtines, Deparment of Chemistry, 10000 Prishtine, Republic of Kosovo. \\ Email: majlinda.ajvazi@uni-pr.edu
}

\section{APPENDIX A}

(supplementary material)

Table of contents

\begin{tabular}{|l|r|}
\hline Figure 1S. Cooxidation of cumene and dodecanal. & Page 2 \\
\hline Experimental procedure for calculations & Page 3 \\
\hline Figures 2S and 3S. Results from calculations. & Page 5 \\
\hline Tables of Cartesian Coordinates & Page 6 \\
\hline Enthalpies obtained from CBS-QB3 calculations & Page 10 \\
\hline
\end{tabular}




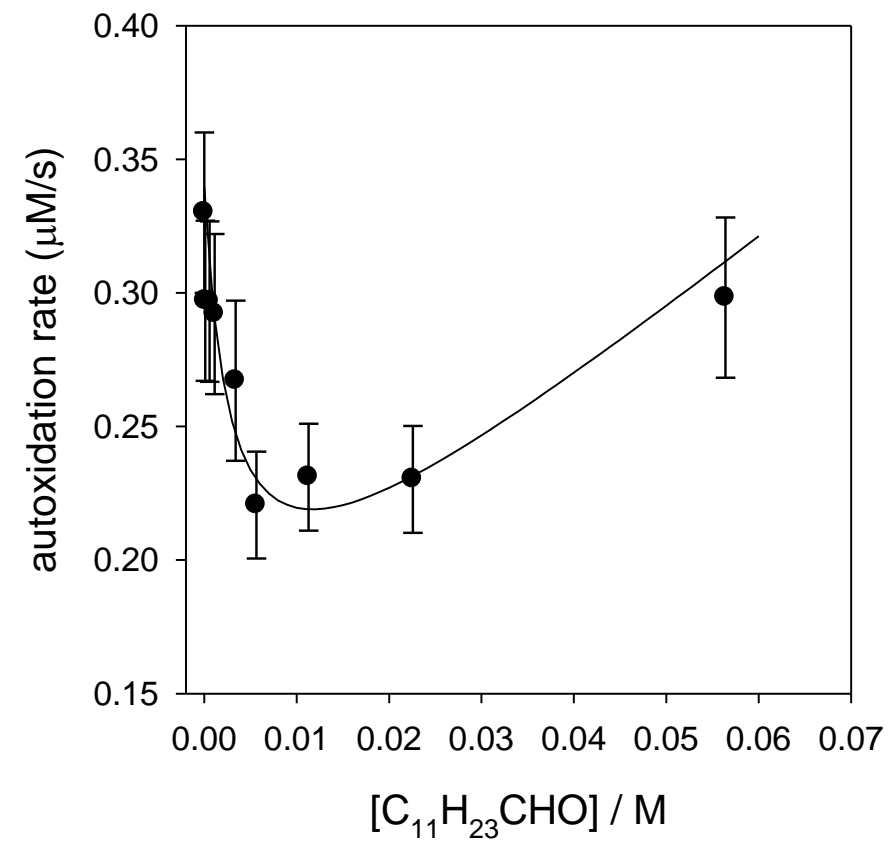

Figure 1S. Rate of $\mathrm{O}_{2}$ uptake during the autoxidation of cumene $(3.5 \mathrm{M})$ in chlorobenzene initiated by $\operatorname{AIBN}(0.05 \mathrm{M})$ at $30^{\circ} \mathrm{C}$, as function of the concentration of dodecanal (range 0.11-56 mM). range. 


\section{Experimental procedure for calculations}

The activation enthalpy of the H-atom transfer from the aldehyde or allylic portion of citral to methylperoxyl radical were computed in the gas phase at the M05/6-311+g(2df,2p) level of theory [1,2], using Gaussian 09 [3]. The hydrocarbon portion of citral was simplified to 2-methyl-2pentene and the aldehydic portion to 3-methyl-2-butenal [4]. Stationary points were confirmed by checking the absence of imaginary frequencies. Transition states had one imaginary frequency corresponding to the transfer of a $\mathrm{H}$-atom. Bond orders for reactants and transition states were computed by using the built-in NBO Gaussian functionality. Orbitals were plotted by using GaussView software (see Figure 2S).

For the sake of comparison, calculations were repeated at the M06-2X/6-311++G(d,p) level of theory[5,6]. The results, summarized in Figure $3 \mathrm{~S}$, qualitatively confirm those calculated at the M05/6-311+g(2df,2p) level.

The bond dissociation enthalpies of the involved species were also computed at the CBS-QB3 level, and were used to calculate the exothermicity of the reaction [7], since this level had previously been shown to afford the most reliable results with similar reactions $[8,9]$.

\section{References}

[1] A. Galano, L. MuÇoz-Rugeles, J. R. Alvarez-Idaboy, J. L. Bao, D. G. Truhlar, J. Phys. Chem. A 2016, 120, 4634- 4642;

[2] O. Tishchenko, D. G. Truhlar, J. Phys. Chem. Lett. 2012, 3, 2834 -2839

[3] Gaussian 09, Revision D.01, Frisch, M. J.; Trucks, G. W.; Schlegel, H. B.; Scuseria, G. E.; Robb, M. A.; Cheeseman, J. R.; Scalmani, G.; Barone, V.; Mennucci, B.; Petersson, G. A.; Nakatsuji, H.; Caricato, M.; Li, X.; Hratchian, H. P.; Izmaylov, A. F.; Bloino, J.; Zheng, G.; Sonnenberg, J. L.; Hada, M.; Ehara, M.; Toyota, K.; Fukuda, R.; Hasegawa, J.; Ishida, M.; Nakajima, T.; Honda, Y.; Kitao, O.; Nakai, H.; Vreven, T.; Montgomery, J. A., Jr.; Peralta, J. E.; Ogliaro, F.; Bearpark, M.; Heyd, J. J.; Brothers, E.; Kudin, K. N.; Staroverov, V. N.; Kobayashi, R.; Normand, J.; Raghavachari, K.; Rendell, A.; Burant, J. C.; Iyengar, S. S.; Tomasi, J.; Cossi, M.; Rega, N.; Millam, J. M.; Klene, M.; Knox, J. E.; Cross, J. B.; Bakken, V.; Adamo, C.; Jaramillo, J.; Gomperts, R.; Stratmann, R. E.; Yazyev, O.; Austin, A. J.; Cammi, R.; Pomelli, C.; Ochterski, J. W.; Martin, R. L.; Morokuma, K.; Zakrzewski, V. G.; Voth, G. A.; Salvador, P.; Dannenberg, J. J.; 
Dapprich, S.; Daniels, A. D.; Farkas, Ö.; Foresman, J. B.; Ortiz, J. V.; Cioslowski, J.; Fox, D. J. Gaussian, Inc., Wallingford CT, 2009.

[4] Hagvall, L.; Backtorp, C.; Norrby, P.-O.; Karlberg, A.-T.; Borje, A. Chem. Res. Toxicol. 2011, $24,1507-1515$.

[5] A. Galano, Phys. Chem. Chem. Phys., 2011, 13, 7178-7188. DOI: 10.1039/c0cp02801k

[6] M. V. La Rocca, M. Rutkowski, S. Ringeissen, J. Gomar, M.-C. Frantz, S. Ngom, C. Adamo J. Mol. Model, 2016, 22, 250. doi:10.1007/s00894-016-3118-6.

[7] Montgomery, J. A.; Frisch, M. J.; Ochterski, J. W.; Petersson, G. A. J. Chem. Phys. 1999, 110, 2822.

[8] R. Amorati, G. F. Pedulli, D. A. Pratt, L Valgimigli. Chem. Commun., 2010, 46, 5139-5141

[9] Z. Zielinski, N. Presseau, R. Amorati, L. Valgimigli, D. A. Pratt J. Am. Chem. Soc. 2014, 136, $1570-1578$ 


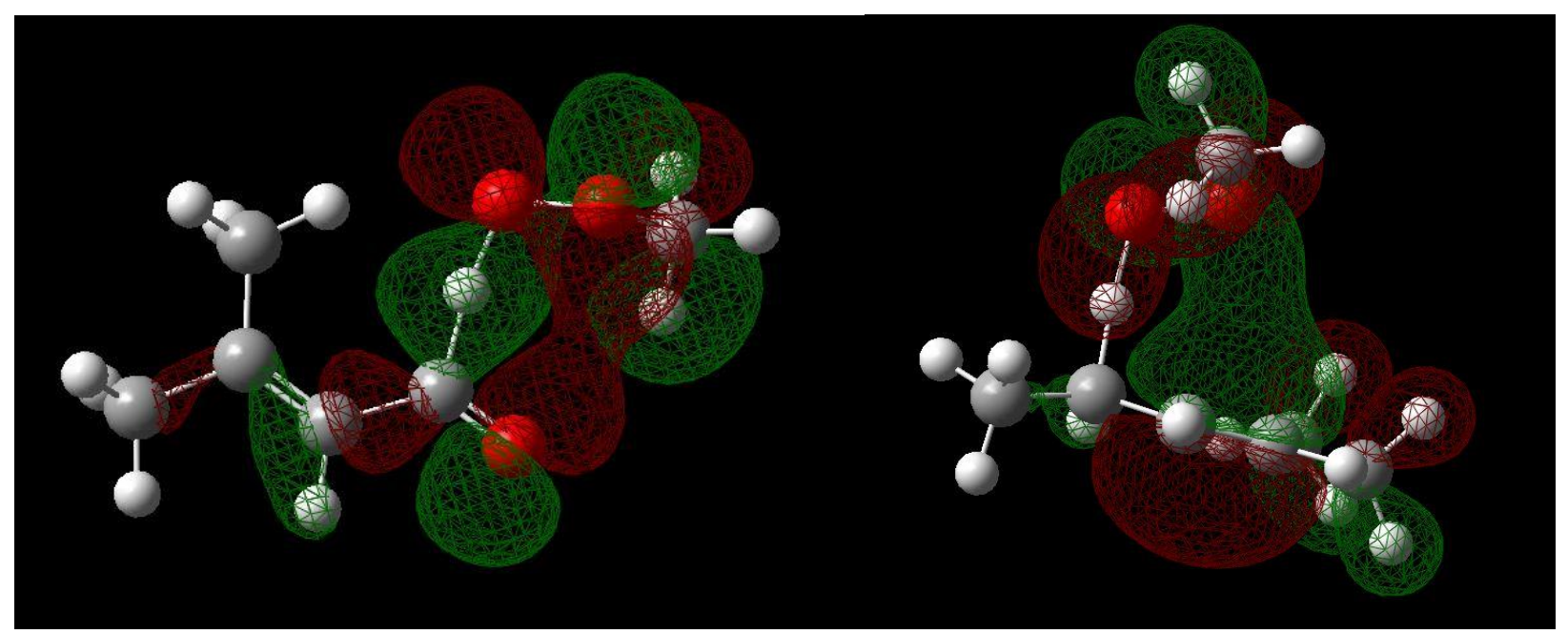

Figure 2S. Plot of SOMO-1 orbitals of the transition states for the reaction at the aldehyde (left) and allylic (right) $\mathrm{CH}$ bond, showing the through-space electron connection between the reactants.

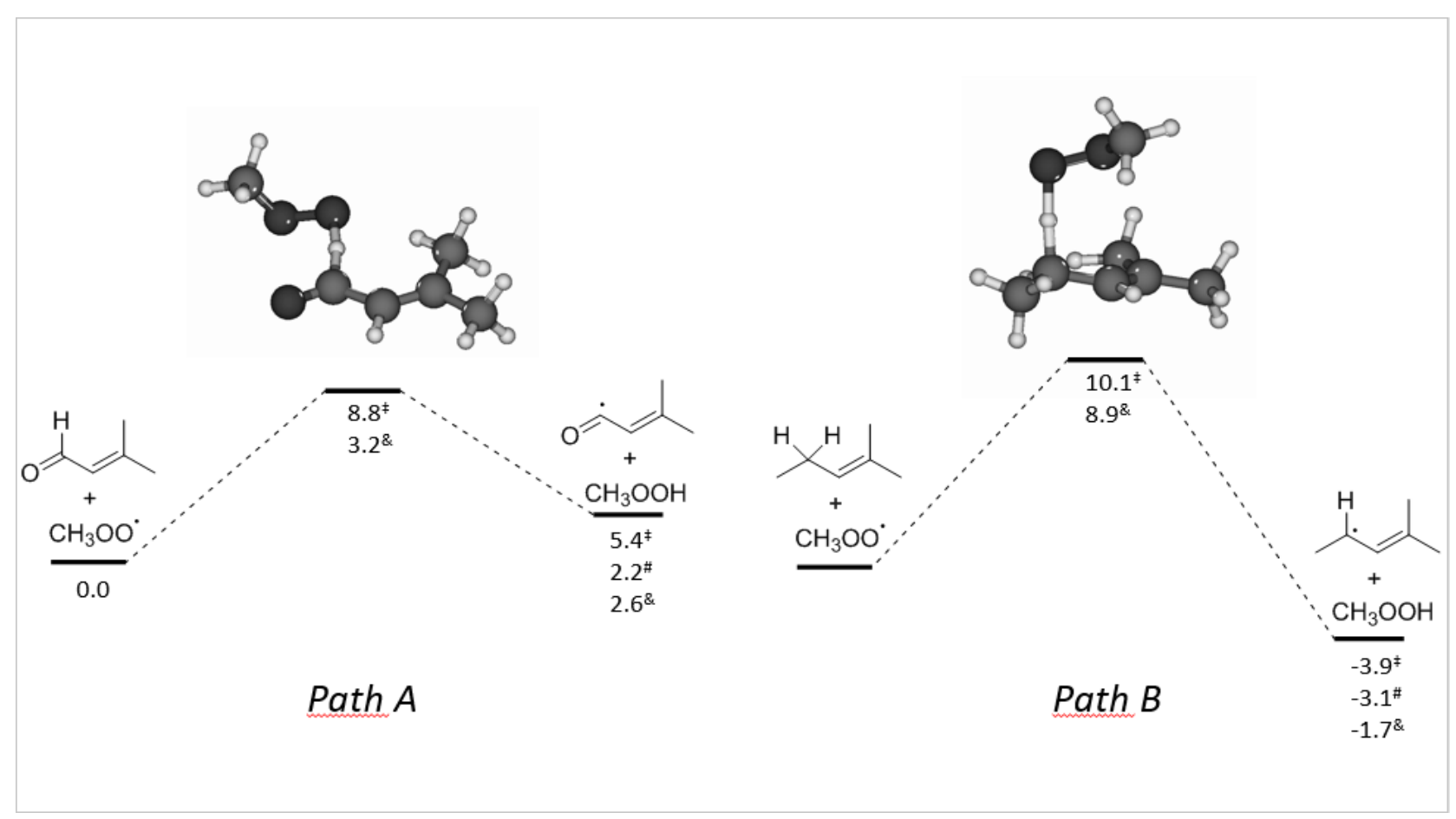

Figure 3S. Reaction profile of $\mathrm{H}$-atom abstraction by ROO radicals from the aldehyde (path A) or allylic (path B) portions of citral, computed at different levels of theory: $(\$)$ M05/6-311+g(2df,2p); (\&) M06-2X/6-311++G(d,p); (\#) CBS-QB3. At each level of theory reported values refer to reactants calculated at the same level of theory. 


\section{Optimized cartesian coordinates.}

\begin{tabular}{|c|c|c|}
\hline structure & M05/6-311+g(2df,2p) level & M06-2X/6-311++G(d,p) \\
\hline & $\begin{array}{l}\text { C,-0.0386775409,-0.2085792758,-0.0152371439 } \\
\text { C,-0.0337818348,-0.0861545804,1.3153082942 } \\
\text { H,0.9277824274,-0.3328007314,--0.4999190371 } \\
\text { C,1.2507388604,-0.130805633,2.0888540554 } \\
\text { C,-1.2568152485,0.0975537932,2.1622941682 } \\
\text { C,-1.1984467255,-0.1837754265,-0.9583664977 } \\
\text { H,1.3971573539,0.7913395493,2.6579480629 } \\
\text { H,1.2400102053,-0.9454147628,2.8183158185 } \\
\text { H,2.113934842,-0.269077968,1.4400357718 } \\
\text { H,-1.1758520533,1.0109368981,2.7573752783 } \\
\text { H,-2.1775535721,0.1543782121,1.5877331339 } \\
\text { H,-1.3566937943,-0.7256716597,2.8748349185 } \\
\text { C,-1.1209754805,0.9678233823,-1.9574181667 } \\
\text { H,-1.2181586816,-1.1268247991,-1.5141191861 } \\
\text { H,-2.1444238457,-0.1332903492,-0.4198197464 } \\
\text { H,-1.9496100844,0.9323238072,-2.6657092158 } \\
\text { H,-1.1536046315,1.9309599289,-1.4468248073 } \\
\text { H,-0.19289491,0.9314408025,-2.5302780765 }\end{array}$ & $\begin{array}{l}\text { C,0.0238135127,-0.3562827801,-0.0541609496 } \\
\text { C,-0.003902519,--0.1044937728,1.2567141512 } \\
\text { H,0.9918869021,-0.5775382889,-0.5013799216 } \\
\text { C,1.2495764681,-0.1534750415,2.0896644901 } \\
\text { C,-1.2465904838,0.2514613978,2.0285135156 } \\
\text { C,-1.1348325857,--0.3217850443,-1.0131938156 } \\
\text { H,1.4391755043,0.8164254996,2.560520946 } \\
\text { H,1.1460011377,-0.883844768,2.8986658042 } \\
\text { H,2.1206764294,--0.4232117093,1.4916014869 } \\
\text { H,-1.1029329182,1.2045068685,2.5472184004 } \\
\text { H,-2.1313994201,0.3408501881,1.4003886226 } \\
\text { H,-1.4450722744,-0.4997633866,2.7998061395 } \\
\text { C,-1.2157397206,1.0152087678,-1.7604875065 } \\
\text { H,-1.0168903398,-1.1319665461,-1.7388196586 } \\
\text { H,-2.0769410049,-0.5106211063,-0.4948910056 } \\
\text { H,-2.0265394336,1.0140371917,-2.4923747117 } \\
\text { H,-1.3854513601,1.8337907379,-1.0573044795 } \\
\text { H,-0.2814714382,1.2208480131,--2.2883675054 }\end{array}$ \\
\hline & $\begin{array}{l}\text { C,0.0043171079,-0.1118446269,0.0274843069 } \\
\text { C,- } 0.0076813805,-0.0292770228,1.5175457206 \\
\text { C,1.1813647215,-0.0773113678,2.2388827965 } \\
\text { C,1.3600783601, }-0.0153968836,3.605799069 \\
\text { C,2.6854421487,--0.0762565062,4.2775632861 } \\
\text { C,-1.3529425149,0.1044557187,2.1476976815 } \\
\text { H,2.0877340852,-0.1767274233, 1.6453761819 } \\
\text { H,-0.5809125173,--0.9667623996,-0.3273659624 } \\
\text { H,-0.4526601779,0.7756571022,--0.4228641711 } \\
\text { H,1.0129595346,-0.2079490646,-0.3715651351 } \\
\text { H,-1.8645110602,1.0013709769,1.7844965982 } \\
\text { H,-1.9934140433,-0.7415639883,1.8791545309 } \\
\text { H,-1.3176140112,0.1607557132,3.2326003899 } \\
\text { H,0.4965700932,0.0838913762,4.2536066012 } \\
\text { H,2.8745784227,0.8211216623,4.8743447559 } \\
\text { H,2.746093157,-0.9212988214,4.9699760066 }\end{array}$ & $\begin{array}{l}\text { C,0.0080043115,0.0533155097,-0.0310178262 } \\
\text { C,- }-0.0165944314,0.0196255159,1.4647233528 \\
\text { C,1.3013702084,0.0092480487,2.1824897952 } \\
\text { C,-1.2176102288,-0.0014095281,2.168910251 } \\
\text { C,-1.3495461925,-0.0327780948,3.5406484946 } \\
\text { C,-2.6662326229,--0.0543701325,4.2472476681 } \\
\text { H,-2.1345209498,0.0077976061,1.5811010372 } \\
\text { H,0.5369635764,0.9420310022,-0.3953442916 } \\
\text { H,0.5446021018,-0.8135197168,--0.4347590817 } \\
\text { H,-0.9981297903,0.0584029963,-0.4526315376 } \\
\text { H,1.4082256805,-0.8844853246,2.8071943201 } \\
\text { H,2.134728707,0.0286675544,1.4786227759 } \\
\text { H,1.4005761823,0.8749536906,2.8466777846 } \\
\text { H,--0.457034128,-0.0427832538,4.159378363 } \\
\text { H,-2.7697531374,-0.9470393749,4.873139898 } \\
\text { H,-2.7773910545,0.8083789801,4.9125475613 }\end{array}$ \\
\hline
\end{tabular}




\begin{tabular}{|c|c|c|}
\hline & $\mathrm{H}, 3.4991015529,-0.1756867007,3.559171401$ & H,-3.4950205832,-0.0420292031,3.5369006016 \\
\hline \multirow{24}{*}[\underbrace{\mathrm{O}_{1}^{-\mathrm{O}^{\prime}}}]{$^{\mathrm{CH}_{3}}$} & C,-0.0801232367,0.2622218814,-0.0184080867 & C,-0.0469507109,0.2706318538,-0.0827346033 \\
\hline & C,--0.0095392483,-0.0041311421,1.3098077023 & $\mathrm{C}, 0.0391650202,-0.1154802681,1.2065457904$ \\
\hline & $\mathrm{H}, 0.8650779539,0.3857995896,-0.5427452161$ & $\mathrm{H}, 0.882728841,0.4599793932,-0.619382656$ \\
\hline & C,1.3091569293,-0.0775181682,2.0092655565 & C,1.3642308068,-0.2229136229,1.9026631741 \\
\hline & C,-1.2039187249,-0.2898715266,2.1596597433 & C,-1.1582988527,-0.4774873374,2.0360347699 \\
\hline & C,-1.2681822762,0.3042479333,-0.8433651834 & $\mathrm{C},-1.2794763347,0.3627120765,-0.862669134$ \\
\hline & $\mathrm{H}, 1.3637544503,0.6572013394,2.8172253464$ & $\mathrm{H}, 1.370909146,0.3828823178,2.8146213707$ \\
\hline & $\mathrm{H}, 1.4439244044,-1.0572920631,2.4764211108$ & $\mathrm{H}, 1.5450432967,-1.2578932158,2.2123423641$ \\
\hline & $\mathrm{H}, 2.1451087267,0.0962962074,1.3340333779$ & $\mathrm{H}, 2.1878560143,0.1002125825,1.265034932$ \\
\hline & $\mathrm{H},-1.1368210032,0.2315681381,3.1167713264$ & H,-1.3559685489,0.3022857444,2.7800298955 \\
\hline & $\mathrm{H},-2.1440945881,-0.0206857021,1.6857450675$ & $\mathrm{H},-2.0534838478,-0.6332485001,1.4361806036$ \\
\hline & $\mathrm{H},-1.249211333,-1.359925078,2.3811151577$ & H,-0.9620914385,-1.4044634054,2.5810492346 \\
\hline & C,-1.1576995567,0.9265175474,-2.2130940931 & C,-1.1611847915,0.9239771087,-2.2636437117 \\
\hline & $\mathrm{H},-1.5423214324,-0.917131002,-1.0197316186$ & H,-1.6528446887,-0.8599241723,-0.9707767932 \\
\hline & $\mathrm{H},-2.1761202099,0.5926874255,-0.3145228608$ & $\mathrm{H},-2.1371157958,0.7474457471,-0.3082652528$ \\
\hline & $\mathrm{H},-2.0444659094,0.7237710176,-2.8127728732$ & H,-2.0904743877,0.7993964481,-2.8211918948 \\
\hline & $\mathrm{H},-1.0461041839,2.0122218844,-2.1462177464$ & $\mathrm{H},-0.9267000993,1.993381033,-2.2373893824$ \\
\hline & $\mathrm{H},-0.2941866855,0.5406505112,-2.7568002362$ & $\mathrm{H},-0.3644245234,0.4219815479,-2.8181210703$ \\
\hline & O,-1.8154777214,-2.1913576325,-1.089549523 & $\mathrm{O},-1.9345351623,-2.1032790958,-0.9050507923$ \\
\hline & O,-0.8433764751,-2.7874352097,-0.3632197937 & O,-0.9284380274,-2.6168872742,-0.1330334872 \\
\hline & $\mathrm{C}, 0.2050049077,-3.2193394385,-1.2136927797$ & C,0.1463730649,-2.9947131161,-0.9765939766 \\
\hline & $\mathrm{H}, 0.9275804479,-3.7094013488,-0.5630234015$ & $\mathrm{H}, 0.9150022777,-3.3969973317,-0.316198642$ \\
\hline & $\mathrm{H},-0.17451233,-3.9232906392,-1.9557028763$ & H,-0.1841877185,-3.7541959532,-1.6879935949 \\
\hline & $\mathrm{H}, 0.6706771688,-2.3688928641,-1.7164303742$ & $\mathrm{H}, 0.5289965346,-2.1204908999,-1.5106894177$ \\
\hline
\end{tabular}




\begin{tabular}{|c|c|c|}
\hline structure & M05/6-311+g(2df,2p) level & M06-2X/6-311++G(d,p) \\
\hline$H$ & $\begin{array}{l}\text { C,-0.0456681219,0.0034322789,-0.0449010838 } \\
\text { C,- } 0.0442505655,0.0231241755,1.2996939751 \\
\text { H,0.8949779443,0.0803679033,-0.5789231136 } \\
\text { C,1.2414576666,0.1498146524,2.0522276523 } \\
\text { C,-1.2604035823,-0.0737738031,2.1658440468 } \\
\text { C,-1.2146685047,-0.1147650891,-0.9127887504 } \\
\text { H,1.2241781456,1.0359057623,2.6914870068 } \\
\text { H,1.3809851669,-0.7063104342,2.7168260247 } \\
\text { H,2.1018291016,0.2176313877,1.3909291876 } \\
\text { H,-1.172393901,-0.93381776,2.8335138795 } \\
\text { H,-1.3290695895,0.8070752959,2.8081877158 } \\
\text { H,-2.19181998,-0.1655521918,1.6172410557 } \\
\text { O,-1.1535835631,-0.1273462157,-2.119695931 } \\
\text { H,-2.1987807234,-0.1957859621,-0.4106416654 }\end{array}$ & $\begin{array}{l}\text { C,-0.0391489239,0.0037982187,-0.0470706568 } \\
\text { C,-0.0421832403,0.0231673807,1.2947578398 } \\
\text { H,0.8997481155,0.0804217478,-0.5876025192 } \\
\text { C,1.2459466933,0.1503226967,2.057694134 } \\
\text { C,-1.263469592,-0.0740378988,2.1668708978 } \\
\text { C,-1.2222505156,-0.1153526909,-0.9098739175 } \\
\text { H,1.2207474162,1.0396834667,2.6947597714 } \\
\text { H,1.3783235891,-0.7103562386,2.720384421 } \\
\text { H,2.1071802475,0.2181535805,1.3941394857 } \\
\text { H,-1.1679122616,-0.9371599416,2.8321025477 } \\
\text { H,-1.3253106161,0.8111823053,2.8065618372 } \\
\text { H,-2.1974126307,-0.166122905,1.6190305084 } \\
\text { O,-1.1543975884,-0.1269501331,-2.1158042246 } \\
\text { H,-2.2070711995,-0.1967495884,-0.4169501248 }\end{array}$ \\
\hline & $\begin{array}{l}\text { C,-0.0171986332,0.2813080191,-0.0091535613 } \\
\text { C,- } 0.0412764665,0.1620999989,1.4779107524 \\
\text { C, } 1.2889106682,0.1538641004,2.1528353082 \\
\text { C,-1.2065147502,0.0694327963,2.1430744222 } \\
\text { C,-1.2880364233,-0.0492475433,3.5886348435 } \\
\text { O,-2.2371555072,--0.1388852038,4.2921037494 } \\
\text { H,-2.1536991675,0.0800906859,1.6066494877 } \\
\text { H,0.4931187418,1.2000422457,-0.307394288 } \\
\text { H,0.5523931048,-0.5423088649,-0.4459084858 } \\
\text { H,- } 1.014657732,0.2815915254,-0.4419215615 \\
\text { H,1.895008319,-0.6739102489,1.7779095296 } \\
\text { H,1.8350535972,1.0698904196,1.9167627959 } \\
\text { H,1.1983116314,0.064759197,3.2320455481 }\end{array}$ & $\begin{array}{l}\text { C,0.0152662288,0.1347702742,-0.0051509217 } \\
\text { C,-0.0157503298,0.0144934397,1.489566065 } \\
\text { C, } 1.3235538904,0.0066456333,2.1641154466 \\
\text { C,-1.1821713022,--0.0779229362,2.1465509544 } \\
\text { C,-1.2512677889,-0.1968098291,3.6074026059 } \\
\text { O,-2.2080024752,-0.2843483095,4.2932901581 } \\
\text { H,-2.1316229078,-0.0680393822,1.6142243816 } \\
\text { H,0.5269564829,1.0571248135,-0.2953710427 } \\
\text { H,0.5869877755,-0.692996238,-0.4349528684 } \\
\text { H,-0.9846484906,0.134950603,-0.4374609504 } \\
\text { H,1.9230257777,-0.824113475,1.780565357 } \\
\text { H,1.8629517142,0.9265348752,1.9202058992 } \\
\text { H,1.2391234802,-0.082491651,3.245296532 }\end{array}$ \\
\hline & $\begin{array}{l}C,-0.1286802151,0.2181525305,0.1876923312 \\
C, 0.0186077516,-0.0538148516,1.6497709083 \\
C, 1.2497419359,-0.1788795315,2.1791290419 \\
C, 1.5761114768,-0.4349748679,3.5765769175 \\
O, 2.6715269187,-0.5940114269,4.0258344711\end{array}$ & $\begin{array}{l}\mathrm{C}, 0,-0.0949781346,0.2352734869,0.205049831 \\
\mathrm{C}, 0,0.0312045925,-0.0564529053,1.6721639277 \\
\mathrm{C}, 0,1.247804721,-0.1859849764,2.2260300717 \\
\mathrm{C}, 0,1.4985596432,-0.4572915074,3.6445642384 \\
\mathrm{O}, 0,2.562742083,-0.6254363323,4.1522436094\end{array}$ \\
\hline
\end{tabular}




\begin{tabular}{|c|c|c|}
\hline 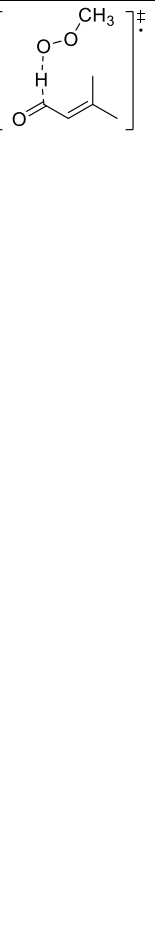 & $\begin{array}{l}\text { C,-1.2677105942,-0.1613053593,2.3983674908 } \\
\text { C,1.1272252153,-0.0173306154,7.2305509435 } \\
\text { O,0.5676540958,-1.0544457313,6.4432467552 } \\
\text { O,-0.1597658874,-0.5244119258,5.4255884161 } \\
\text { H,2.1221914029,--0.0878430457,1.5391266307 } \\
\text { H,-0.6780556002,-0.5949272832,-0.2921551755 } \\
\text { H,-0.7193289212,1.1232882608,0.0296147337 } \\
\text { H,0.8280861842,0.3341433074,-0.3151018385 } \\
\text { H,-1.7738006538,0.8071775343,2.3880991849 } \\
\text { H,-1.9329717577,-0.8570619962,1.8831225325 } \\
\text { H,-1.1664128272,-0.4844015169,3.4276781753 } \\
\text { H,0.6102641902,-0.47504514,4.4689975045 } \\
\text { H,1.650260136,-0.5231217745,8.0401078545 } \\
\text { H,1.8344160908,0.5736837756,6.6457797206 } \\
\text { H,0.3404851046,0.6239718765,7.6311309653 }\end{array}$ & $\begin{array}{l}\text { C,-1.2768064151,-0.1712599813,2.3998782382 } \\
\text { C, } 1.1727617649,0.0078667533,7.1406764315 \\
\text { O,0.6068232865,-1.077329652,6.4215344088 } \\
\text { O,-0.2423773876,-0.583714393,5.469011274 } \\
\text { H,2.1450356561,-0.0895531575,1.6206460869 } \\
\text { H,-0.6163519943,-0.5876370557,-0.2927843911 } \\
\text { H,-0.7031900968,1.1319492008,0.0541711638 } \\
\text { H,0.8733944203,0.378850016,-0.2723739393 } \\
\text { H,-1.7498741921,0.8148119689,2.4424238569 } \\
\text { H,-1.9494625162,-0.8174029211,1.829621473 } \\
\text { H,-1.1979675686,-0.5620948827,3.4108066652 } \\
\text { H,0.4965359969,-0.4968019669,4.4585221844 } \\
\text { H,1.8106384463,-0.447944888,7.8972629051 } \\
\text { H,1.7723527057,0.6312591607,6.4737625709 } \\
\text { H,0.3829990345,0.597736252,7.6099469569 }\end{array}$ \\
\hline
\end{tabular}

\begin{tabular}{|c|c|c|}
\hline structure & M05/6-311+g(2df,2p) level & M06-2X/6-311++G(d,p) \\
\hline & $\begin{array}{l}\text { O,-0.073399014,0.0890928529,-0.0028199348 } \\
\text { C,-0.0393442106,0.0250012404,1.4032772055 } \\
\text { H,1.0207815122,0.0317965031,1.653504671 } \\
\text { H,-0.5316563908,0.8886176424,1.8593160274 } \\
\text { H,-0.4973002781,-0.8967915491,1.7673106061। } \\
\text { O,-1.4209090244,0.0094049894,-0.407735736। } \\
\text { H,- } 1.5803568807,0.8970964487,-0.7474874952\end{array}$ & $\begin{array}{l}\text { O,-0.0847696294,0.1124427797,0.0251591288 } \\
\text { C,-0.0233905578,0.000223934,1.4295231669 } \\
\text { H,1.0412226132,0.0017916115,1.6649658837 } \\
\text { H,-0.5187713039,0.8467495816,1.9123675844 } \\
\text { H,-0.4766581977,-0.9372580431,1.7602401144 } \\
\text { O,-1.4677814373,0.0455755949,-0.3161452343 } \\
\text { H,- } 1.5581796375,0.8233147039,-0.8789995328\end{array}$ \\
\hline $\mathrm{CH}_{3} \mathrm{OO}$ & $\begin{array}{l}\text { O,-0.0599451116,0.,-0.0111813346 } \\
\text { C,-0.0215433411,0., } 1.4298586015 \\
\text { H,1.0329434194,0.,1.6915847588 } \\
\text { H,-0.5173692876,0.8950579968,1.799521599 } \\
\text { H,-0.5173692876,-0.8950579968,1.799521599 } \\
\text { O,-1.2673478499,0.,-0.4649460347 }\end{array}$ & $\begin{array}{l}\text { O,-0.0611965268,0.,-0.0165773634 } \\
\text { C,-0.0268674869,0.,1.4186634375 } \\
\text { H,1.0267559526,0.,1.6888578798 } \\
\text { H,-0.5271209304,0.8964975298, 1.7829637722 } \\
\text { H,-0.5271209304,-0.8964975298, 1.7829637722 } \\
\text { O,-1.2798074596,0.,-0.4612048316 }\end{array}$ \\
\hline
\end{tabular}


CBS-QB3 enthalpies (in Hartree)

\begin{tabular}{|l|l|}
\hline & -235.321269 \\
\hline & -234.691381 \\
\hline & -269.673976 \\
\hline $\mathrm{CH}_{3} \mathrm{OOH}$ & -190.589540 \\
\hline $\mathrm{CH}_{3} \mathrm{OO}$ & -189.954725 \\
\hline $\mathrm{H}^{\circ}$ & -0.497457 \\
\hline
\end{tabular}

